\title{
TNF superfamily receptor OX40 triggers invariant NKT cell pyroptosis and liver injury
}

\author{
Peixiang Lan, ${ }^{1}$ Yihui Fan, ${ }^{1}$ Yue Zhao, ${ }^{1}$ Xiaohua Lou, ${ }^{1}$ Howard P. Monsour, ${ }^{2}$ Xiaolong Zhang, ${ }^{1}$ Yongwon Choi, ${ }^{3}$ Yaling Dou, ${ }^{1}$ \\ Naoto Ishii, ${ }^{4}$ Rafik M. Ghobrial, ${ }^{1,5}$ Xiang Xiao, ${ }^{1}$ and Xian Chang Li ${ }^{1,5}$ \\ IImmunobiology and Transplant Science Center, Houston Methodist Research Institute, and ²Department of Medicine, Division of Hepatology, Houston Methodist Hospital, Texas Medical Center, Houston, \\ Texas, USA. ${ }^{3}$ University of Pennsylvania School of Medicine, Philadelphia, Pennsylvania, USA. ${ }^{4}$ Department of Microbiology and Immunology, Tohoku University School of Medicine, Sendai, Japan. \\ ${ }^{5}$ Department of Surgery, Weill Cornell Medical College of Cornell University, New York, New York, USA.
}

\begin{abstract}
Tissue-resident immune cells play a key role in local and systemic immune responses. The liver, in particular, hosts a large number of invariant natural killer T (iNKT) cells, which are involved in diverse immune responses. However, the mechanisms that regulate survival and homeostasis of liver iNKT cells are poorly defined. Here we have found that liver iNKT cells constitutively express the costimulatory TNF superfamily receptor $\mathrm{OX} 40$ and that $\mathrm{OX} 40$ stimulation results in massive pyroptotic death of iNKT cells, characterized by the release of potent proinflammatory cytokines that induce liver injury. This 0X40/NKT pyroptosis pathway also plays a key role in concanavalin A-induced murine hepatitis. Mechanistically, we demonstrated that liver iNKT cells express high levels of caspase 1 and that OX40 stimulation activates caspase 1 via TNF receptor-associated factor 6 -mediated recruitment of the paracaspase MALT1. We also found that activation of caspase 1 in iNKT cells results in processing of pro-IL-1 $\beta$ to mature IL-1 $\beta$ as well as cleavage of the pyroptotic protein gasdermin D, which generates a membrane pore-forming fragment to produce pyroptotic cell death. Thus, our study has identified $0 \times 40$ as a death receptor for iNKT cells and uncovered a molecular mechanism of pyroptotic cell death. These findings may have important clinical implications in the development of 0X40-directed therapies.
\end{abstract}

\section{Introduction}

A substantial number of immune cells reside in tissues other than the lymphoid compartments, and such tissue-resident immune cells are increasingly appreciated as key players in local immunity and immunopathology, as well as in regulation of systemic immune responses $(1,2)$. The liver in particular is a unique site where diverse immune cell types reside, especially invariant NKT (iNKT) cells. In fact, iNKT cells are a dominant cell type in the liver (1). Of note, several unique features distinguish iNKT cells from conventional T cells; they express an invariant TCR consisting of an invariant $\alpha$ chain (V $14 \mathrm{~J} \alpha 18$ in the mouse, V $\alpha 24 \mathrm{~J} \alpha 18$ in humans) paired with a restricted number of $\beta$ chains, which specifically recognizes lipid antigens presented by CD1d molecules (3). Phenotypically, most iNKT cells are $\mathrm{CD}^{+}{ }^{+} \mathrm{T}$ cells and show features of memory cells, and functionally, iNKT cells are innate-like lymphocytes that can rapidly produce a plethora of pro- and antiinflammatory cytokines upon activation, which affects the induction as well as the outcome of immune responses locally in the liver or systemically outside the liver $(2,4-6)$. Because of that, they are involved in diverse immune responses, ranging from liver injury and regeneration to regulation of adaptive immunity and immunopathology (7-10). However, despite their importance, the exact mechanisms that regulate iNKT cell homeostasis in vivo, as well as their in vivo fates (life and death), are largely unknown.

Conflict of interest: The authors have declared that no conflict of interest exists. Submitted: October 6, 2016; Accepted: February 23, 2017.

Reference information: / Clin Invest. 2017;127(6):2222-2234.

https://doi.org/10.1172/JCI91075.
One intriguing area is that the liver usually provides a tolerogenic milieu for intrahepatic immune cells in spite of a portal drainage, which is rich in food products, antigenic metabolites, and constituents of gut microbiota (11). However, iNKT cells do exhibit features of activation and express surface markers of memory cells (12). The expression of OX40 (also called CD134) by iNKT cells is particularly interesting, as OX40 is a costimulatory molecule in the TNF receptor (TNFR) superfamily and typically associated with $\mathrm{T}$ cell activation (13). Traditionally, OX40 promotes $\mathrm{T}$ cell survival, effector differentiation, and memory generation, especially for $\mathrm{CD} 4^{+} \mathrm{T}$ cells (14). It has been well established that activation of $\mathrm{CD}^{+} \mathrm{T}$ cells in the presence of OX40 costimulation sustains BCL2 expression, leading to the survival advantage of $\mathrm{CD}^{+} \mathrm{T}$ cells and development of long-lived memory cells (15). Conversely, viral infections in OX40-deficient mice result in markedly reduced proliferation of $\mathrm{CD} 4^{+} \mathrm{T}$ cells and impaired generation of virus-specific $\mathrm{CD} 4^{+}$memory $\mathrm{T}$ cells (16). In certain models, OX40 costimulation supports robust Th2 responses and allergic inflammation (17). We reported that OX40 is remarkably potent in facilitating the induction of Th9 cells and airway inflammation, and OX40 accomplishes these effects through activation of the noncanonical NF- $\kappa$ B pathway (18). OX40 costimulation also inhibits Foxp $3^{+}$Tregs $(19,20)$, thus relieving $\mathrm{T}$ effector cells from Treg-mediated suppression, which indirectly boosts $\mathrm{T}$ effector responses. On the other hand, OX40 also contributes substantially to multiple autoimmune diseases. For example, all OX40 ligand-transgenic (OX4OL-Tg) mice, in which the ligand for OX40 is constitutively expressed, spontaneously develop systemic autoimmune diseases characterized by production of auto- 
antibodies and severe inflammation in the gut, liver, and lungs $(21,22)$. Moreover, OX40 costimulation has been linked to the pathogenesis of autoimmune colitis, arthritis, experimental autoimmune encephalomyelitis (EAE), asthma, and tissue fibrosis (13, 23). It has always been assumed that $\mathrm{T}$ cells are the sole effector cells in OX40-mediated pathologies.

Here, we examined the role of liver-resident iNKT cells in OX40-induced responses, focusing on cell survival and memory features, and found unexpectedly that OX40 engagement induced massive pyroptotic death of NKT cells - in that dying NKT cells release potent inflammatory cytokines and induce prominent liver injury - thus establishing OX40 as a death receptor for iNKT cells. We also found that OX40 signaling activates caspase 1 in iNKT cells through recruitment of MALT1, which leads to IL-1 $\beta$ processing, as well as activation of the pyroptotic protein gasdermin $\mathrm{D}$ to induce pyroptotic cell death.

\section{Results}

OX4O stimulation depletes iNKT cells in vivo. We observed before that prolonged stimulation of the OX40/OX40L pathway induces systemic autoimmunity, with prominent involvement of the liver (22). To determine whether liver-resident iNKT cells may have any roles in the liver pathology, we used the CD1d- $\alpha$ GalCer tetramer, which binds to the invariant TCR on iNKT cells (2), to track iNKT cells in naive C57BL/6 (B6) mice. We found that iNKT cells in the liver constitutively express OX40, whereas OX40 is conspicuously absent on other cell types in the liver (Figure 1A), an observation that has also been reported by others (24-26). To determine whether OX40 has any roles in regulation of iNKT cells in the liver, we analyzed iNKT cells, using the same CD1d- $\alpha \mathrm{Gal}-$ Cer tetramer, in the liver of age-matched naive WT B6, OX40L$\mathrm{Tg}$, and $\mathrm{O} x 40-\mathrm{KO}$ mice. As shown in Figure 1B, the iNKT cells accounted for $\sim 6 \%$ of all lymphocytes retrieved from the liver of WT B6 mice. To our surprise, the iNKT cells in OX40L-Tg mice were markedly depleted $(\sim 0.6 \%)$, whereas those in $0 x 40-\mathrm{KO}$ mice were increased by $\sim 2$-fold to about $11.6 \%$ as compared with those in WT B6 mice (Figure 1, B and C). The absolute number of iNKT cells showed similar changes in those mice (Figure 1C). Interestingly, iNKT cells in the thymus did not show differences among those strains (Supplemental Figure 1; supplemental material available online with this article; https://doi.org/10.1172/ JCI91075DS1), suggesting that depletion of iNKT cells in OX4OL$\mathrm{Tg}$ mice is not due to altered iNKT development in the thymus.

In a different set of experiments, we injected an agonist antiOX40 mAb (clone OX86) into WT B6 mice and examined changes in iNKT cells in the liver. As shown in Figure 1D, OX86 also induced marked depletion of iNKT cells in the liver, while blocking the OX40/OX40L pathway by use of an anti-OX40L mAb led to a reciprocal increase in iNKT cells (by $\sim 2$-fold as compared with control IgG-treated mice), both in relative percentage and in absolute cell number (Figure 1E). We did not observe altered distribution of iNKT cells outside of the liver, in locations such as the spleen, thymus, lungs, bone marrow, and lymph nodes, following OX86 treatment (Figure 1F), suggesting that deletion of the intrahepatic iNKT cells by OX40 stimulation is unlikely due to redistribution of iNKT cells in vivo. To ascertain that depletion of iNKT cells is a peripheral event, we FACS-sorted iNKT cells from DsRed mice, in which all
iNKT cells are genetically marked by the red fluorochrome DsRed (27), and adoptively transferred the cells into WT B6 and OX4OL$\mathrm{Tg}$ mice $\left(10^{6}\right.$ cells per mouse). Accumulation of DsRed iNKT cells in the host liver was monitored over time. As shown in Figure 1G, the DsRed iNKT cells were readily identified and remained stable in the liver of WT B6 host mice for more than 2 weeks. In contrast, those in OX4OL-Tg mice were completely depleted 2 weeks after transfer. The use of DsRed as a marker to track iNKT cells also ruled out the possibility that downregulation of TCR, as seen in other conditions, accounted for the absence of iNKT cells in vivo after OX40 stimulation. We did not detect significant DsRed iNKT cells in other compartments in either the WT B6 mice or the OX40L-Tg mice after cell transfer (data not shown). Collectively, these data suggest that the OX40/OX40L pathway plays an important role in life and death of iNKT cells in vivo.

Depletion of $i N K T$ cells in vivo requires caspase 1 activation. We reasoned that OX40 may trigger the demise of iNKT cells in vivo, e.g., through the activation cell death pathways. So we first examined induction of key inflammatory as well as apoptotic caspases, which often mediate different forms of cell death, by OX40 in iNKT cells (28-30). As shown in Figure 2A, freshly isolated iNKT cells from the liver constitutively expressed caspase 1 and caspase 3 , as revealed by immunoblotting assays. As compared with naive $\mathrm{T}$ cells and NK cells, the expression of caspase 1 is confined to iNKT cells, whereas caspase 3 is expressed not only in iNKT cells but also in resting $\mathrm{T}$ cells and NK cells (Figure 2A). To determine the roles of caspases 1 and 3, which require proteolytic cleavage to become active (31), in the life and death of iNKT cells in response to OX40 stimulation, we FACS-sorted iNKT cells from naive $\mathrm{B} 6$ mice and stimulated them in vitro with different doses of OX86; we then examined cleavage of caspase $1 / 3$ by immunoblotting 30 minutes later. As shown in Figure 2B, stimulation of OX40 on iNKT cells triggered robust cleavage of caspase 1, resulting in the formation of a $20-\mathrm{kDa}$ active caspase 1 fragment from its $\sim 45$-kDa precursor. In contrast, cleavage of caspase 3 was not observed regardless of OX40 stimulation (Figure 2B). Moreover, activation of caspase 1 in iNKT cells in response to OX40 stimulation was further confirmed by flow cytometry using a staining $\mathrm{Ab}$ that specifically recognizes cleaved caspase 1 (Figure $2 \mathrm{C}$ ). When compared with control IgG-treated iNKT cells, as much as $25 \%$ of iNKT cells stained positive for the cleaved caspase 1 at 24 hours after OX40 stimulation, and this cleaved caspase 1-positive proportion increased to $\sim 35 \%$ at 48 hours (Figure $2 \mathrm{C}$ ).

To address the possible role of caspase 1 in iNKT cell depletion, we took the following three approaches. First, we treated naive B6 mice with the agonist anti-OX40 mAb OX86; groups of treated mice were also given a caspase 1 inhibitor (or a caspase 3 inhibitor as controls), and the intrahepatic iNKT cells were assessed using the CD1d- $\alpha$ GalCer tetramer. We observed that treatment with the caspase 1 inhibitor rescued iNKT cells from OX40-induced depletion, whereas the caspase 3 inhibitor failed to do so (Figure 2D). Second, we tested the same caspase inhibitors in OX40L-Tg mice, in which iNKT cells are absent (Figure 1B), asking whether caspase 1 inhibition would rescue iNKT cells. As shown in Figure 2E, treatment of OX4OL-Tg mice with the caspase 1 inhibitor completely rescued iNKT cells from depletion in vivo, but the caspase 3 inhibitor had no effect. Third, we treated 

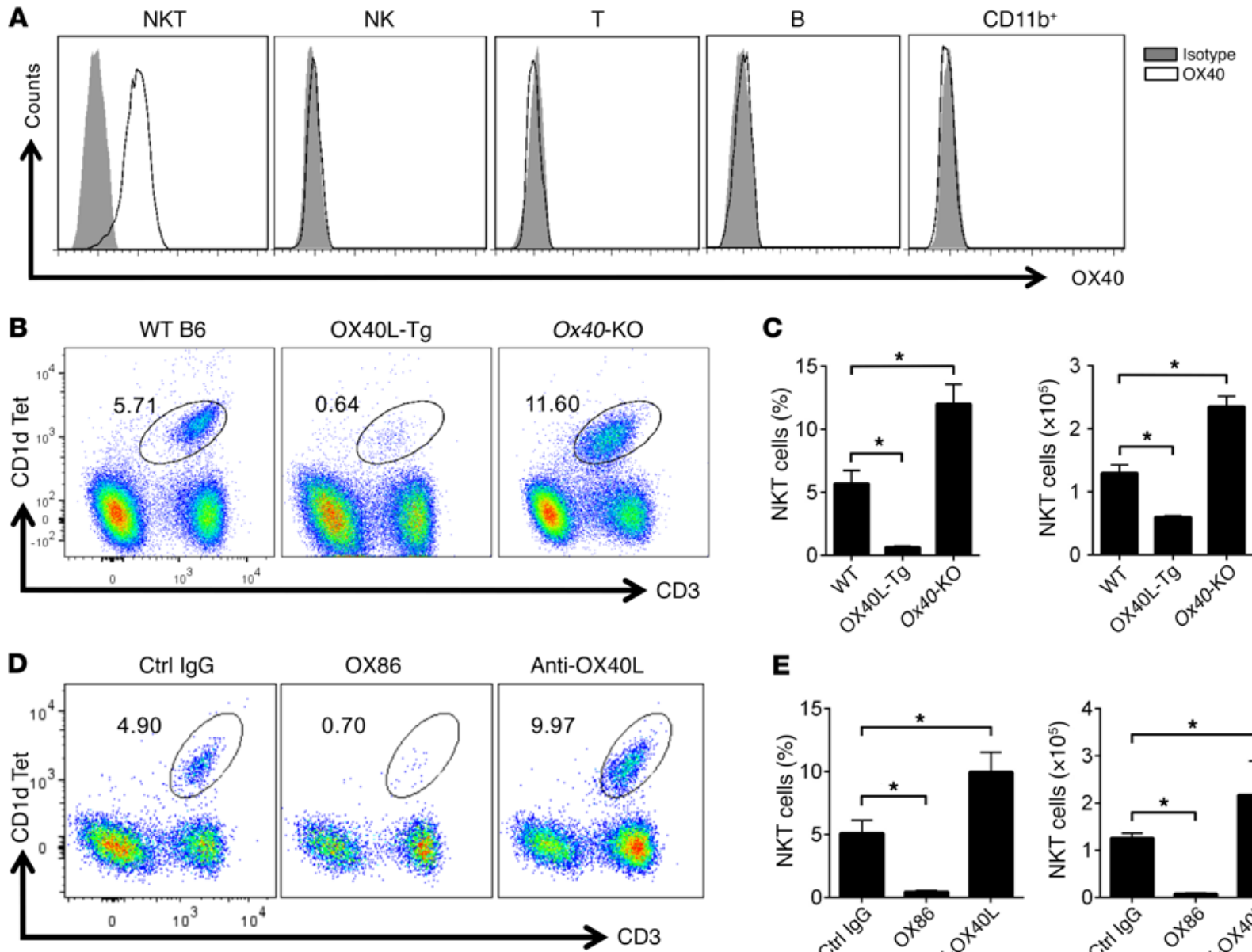

E
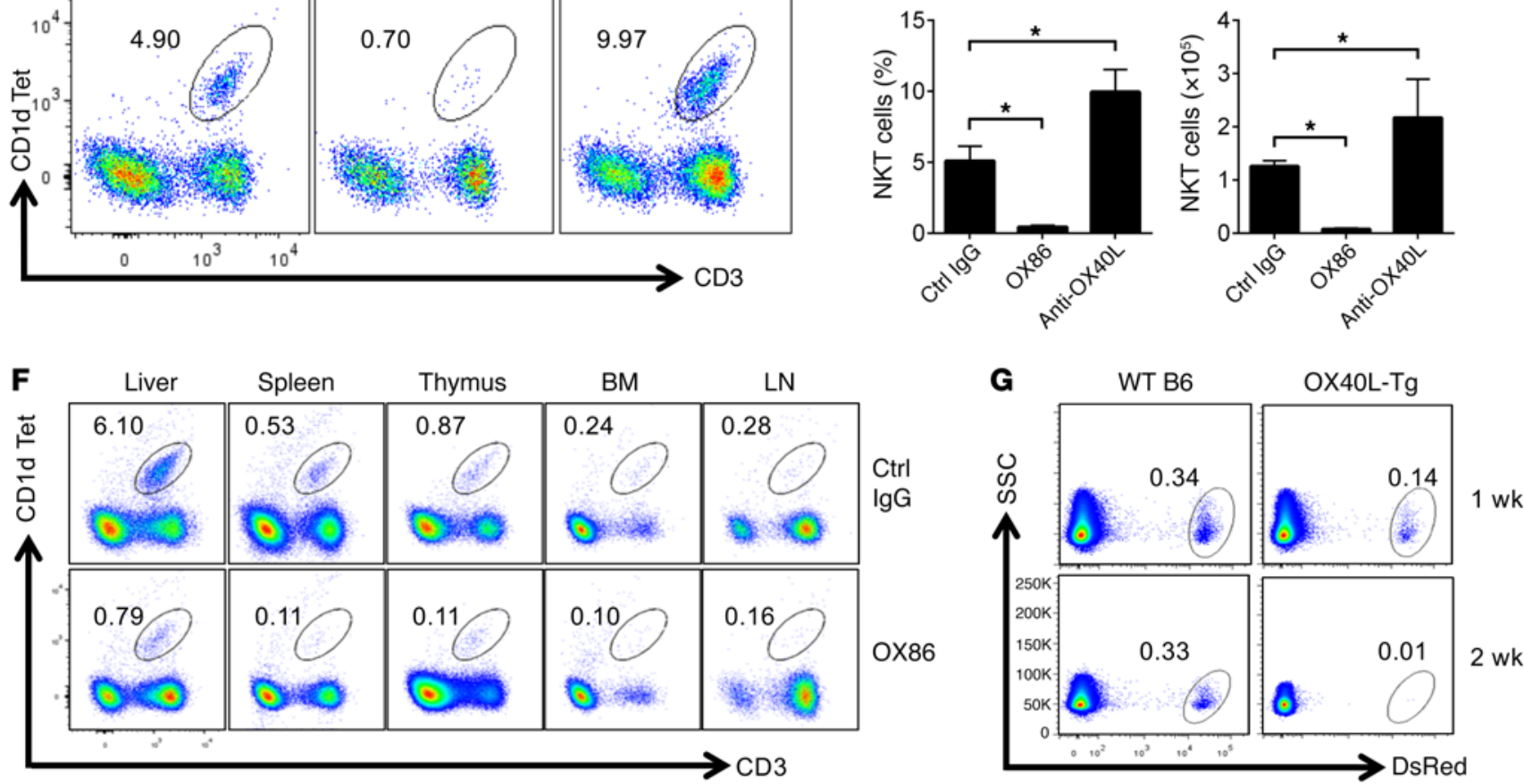

Figure 1. OX40 stimulation readily depletes iNKT cells in vivo. (A) Cell surface expression of OX40 by iNKT, NK, T, and B cells and CD11b+ cells from the liver of naive WT B6 mice was analyzed by FACS. Data shown are from one of 3 independent experiments. (B) Mononuclear cells in the liver of WT B6, OX4OL-Tg, and Ox4O-KO mice were isolated, and iNKT cells were identified by CD1d- $\alpha$ CalCer and CD3 staining and analyzed by FACS. The gate shows iNKT cells, as well as the relative percentage among total cells retrieved. The FACS plots shown represent one of 6 individual experiments. (C) The summary bar graphs represent relative percentage and absolute number of iNKT cells in the liver of WT B6, OX40L-Tg, and Ox4O-KO mice. The data shown are mean \pm SD of 6 experiments. (D) The FACS plot shows the relative percentage of iNKT cells in the liver of WT B6 mice 2 weeks after treatment with OX86, anti-OX40L mAb, or a control (Ctrl) IgG (200 $\mu$ g, i.p.). The plot shown represents one of 6 individual experiments. (E) The summary graphs show relative percentage and absolute number of iNKT cells from the liver of WT B6 mice treated with OX86, anti-OX40L mAb, or control lgG. Data shown are mean \pm SD of 6 experiments. (F) WT B6 mice were treated with OX86 or control lgG (200 $\mu$ g, i.p.), and iNKT cells in the host spleen, thymus, bone marrow (BM), and lymph nodes (LN) were determined using CD1d- $\alpha$ CalCer and anti-CD3 staining, gated, and analyzed by FACS. Data shown represent one of 3 individual experiments. (C) FACS-sorted iNKT cells from the liver of DsRed mice were transferred into WT B6 or OX40L-Tg hosts $\left(1 \times 10^{6}\right.$ cells per mouse). The presence of DsRed iNKT cells in the liver of host mice was determined by FACS. Data shown represent one of 3 independent experiments. $P$ values were calculated using 1-way ANOVA (C and $\mathbf{E}),{ }^{*} P<0.05$ 
A

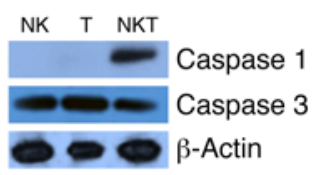

B

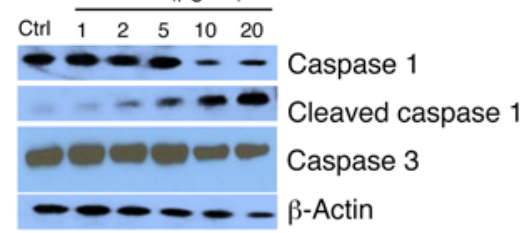

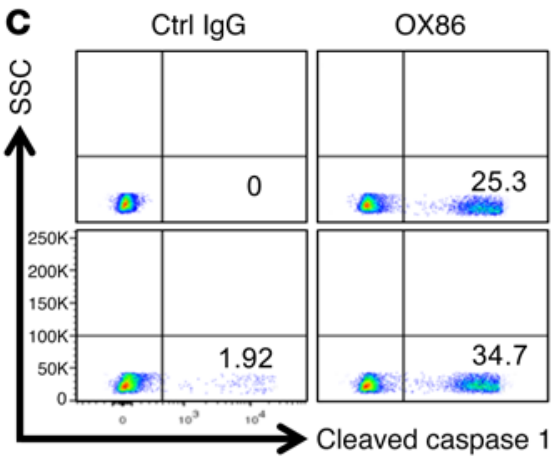

$24 \mathrm{~h}$

E

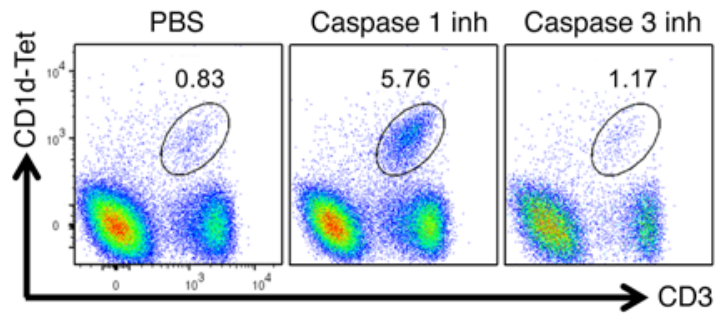

H

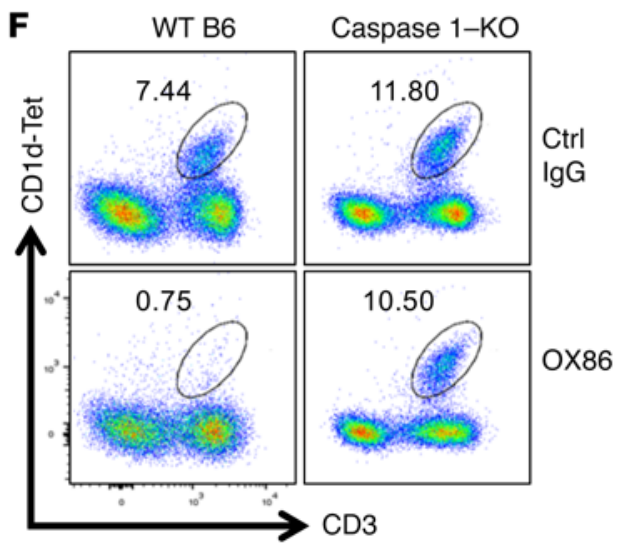

G

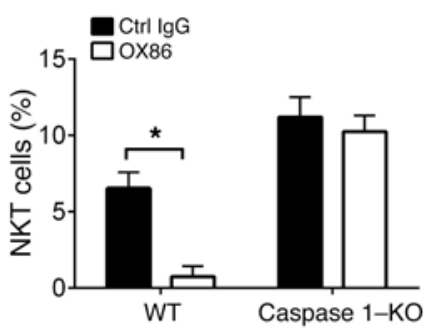

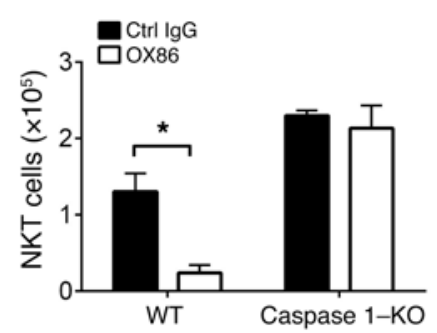

Figure 2. Critical role of caspase 1 in $\mathbf{0 X 4 0}$-induced depletion of iNKT cells. (A) Immunoblot analysis of caspase 1 and caspase 3 in liver iNKT, NK, and T cells from WT B6 mice. $\beta$-Actin was used as a loading control. The blot shown is representative of one of 6 experiments. (B) FACS-sorted iNKT cells from WT B6 mice were stimulated with 0X86 for 30 minutes, and cleavage of caspase 1 and caspase 3 was determined by immunoblotting. $\beta$-Actin was used as a loading control. The blot shown is representative of one of 6 experiments. (C) WT B6 mice were treated with either OX86 or control IgG (200 $\mu$ g, i.p.). Caspase 1 activation in liver iNKT cells was determined using a specific Ab that stains the cleaved active caspase 1 fragment 24 and 48 hours later. The FACS shown is representative of one of 6 individual experiments. (D) WT B6 mice were treated with 0 X86 (200 $\mu$ g, i.p.), and groups of mice were also treated with PBS as a control, caspase 1 inhibitor (inh), or caspase 3 inhibitor $(1 \mathrm{mg} / \mathrm{kg}$, i.p.). The iNKT cells in the liver of treated mice were determined by FACS 2 weeks later. The FACS plot shown represents data for one of 10 animals. (E) Six-week-old OX40L-Tg mice were treated with the caspase 1 inhibitor or caspase 3 inhibitor (1 mg/kg), or PBS as a control. The iNKT cells in the liver of treated mice were determined by FACS 2 weeks later. The FACS plot shown is representative data of one of 10 animals. (F) WT B6 mice and caspase 1-KO mice were treated with OX86 or a control IgC (200 $\mu \mathrm{g}$, i.p.), and iNKT cells in liver of the treated mice were analyzed by FACS 2 weeks later. Data shown are from one of 6 independent experiments. (G and $\mathbf{H})$ The summary bar graphs show relative percentage and absolute number of iNKT cells in WT B6 and caspase 1-KO mice with or without OX86 treatment. Data shown are mean \pm SD of 6 experiments. $P$ values were calculated by unpaired 2-tailed Student's $t$ test between control and $0 \times 86$-treated groups $(\mathbf{G}$ and $\mathbf{H}),{ }^{*} P<0.05$.

WT B6 and caspase 1-KO mice with the same doses of OX86 and then compared the survival of iNKT cells in the host liver 2 weeks later. We found that there was a substantial increase in iNKT cells in caspase 1-KO mice as compared with age-matched WT B6 mice (Figure $2 \mathrm{~F}$ ), in both relative percentage (Figure 2G) and absolute cell numbers (Figure $2 \mathrm{H}$ ). While OX86 treatment readily depleted iNKT cells in WT B6 mice, treatment of caspase 1-KO mice with OX86 failed to deplete iNKT cells in vivo, and a similar number of iNKT cells was observed in caspase 1-KO mice with or without
OX86 treatment (Figure 2, F-H). Collectively, these data provide strong evidence that caspase 1 is required for depletion of iNKT cells in vivo in response to OX40 stimulation.

iNKT cells die of pyroptosis upon OX4O stimulation. Caspase 1 is best known for converting pro-IL-1 $\beta$ and pro-IL-18 to their biologically active forms, and in macrophages caspase 1 is also instrumental in generating the $\mathrm{N}$-domain fragment from gasdermin $\mathrm{D}$ (GSDMD), which is a powerful membrane pore-forming molecule in pyroptotic cell death $(32,33)$. We challenged WT B6 mice with 
A

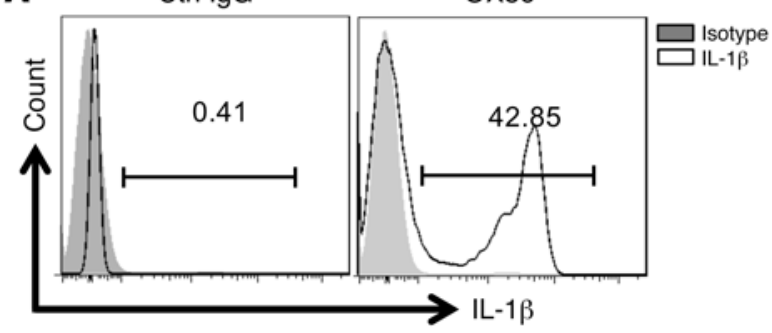

B
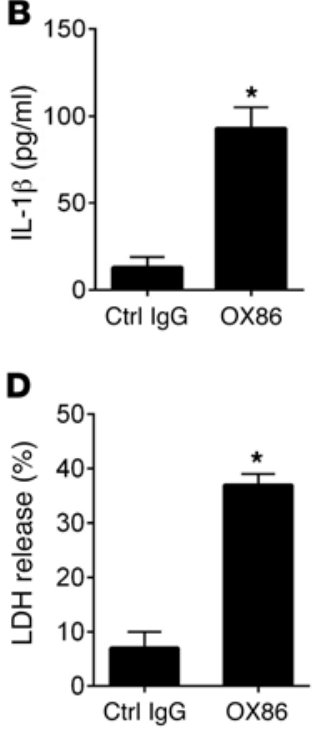

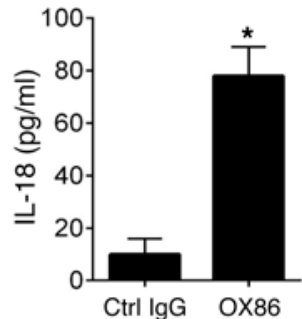

E

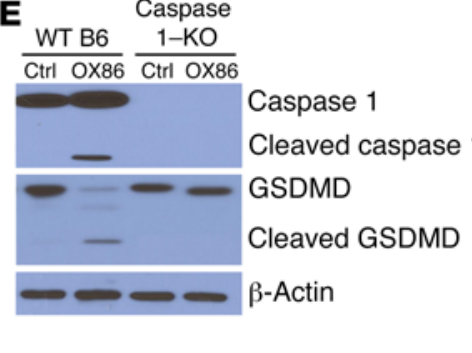

C
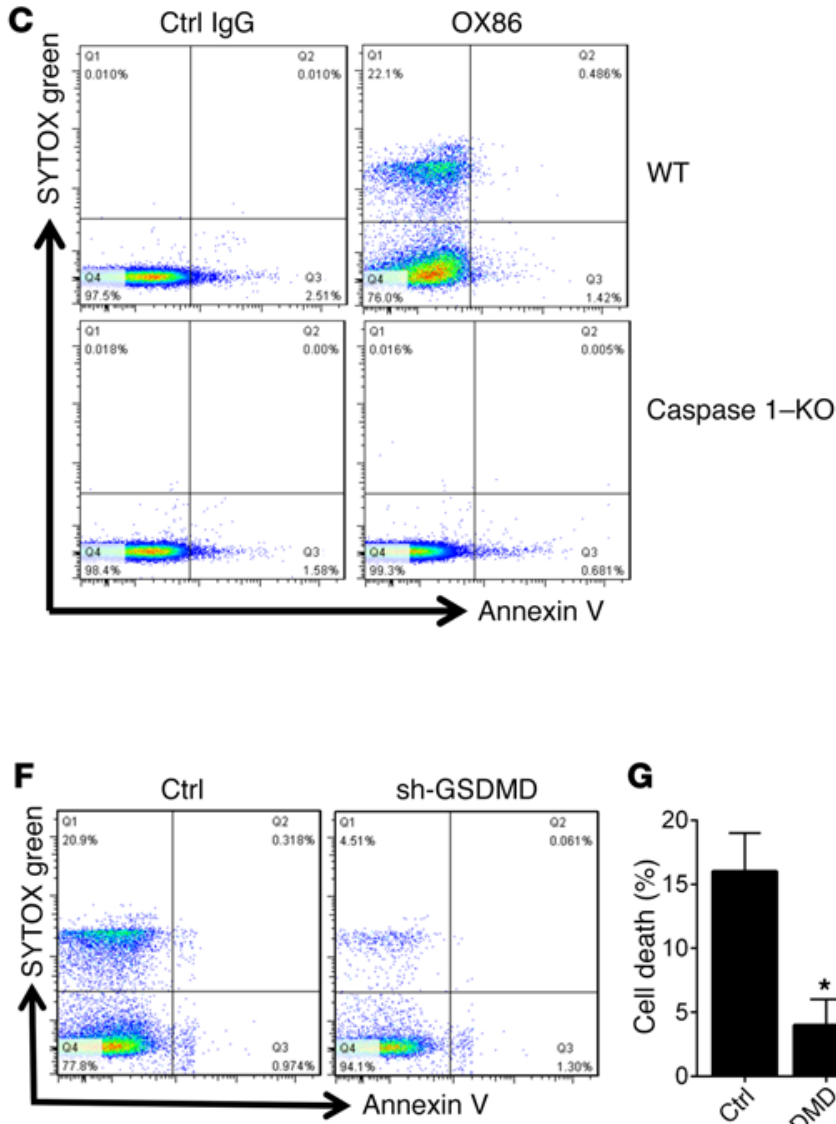

G

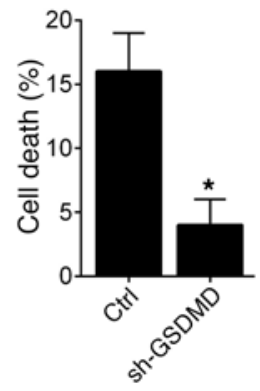

Figure 3. Involvement of GSDMD cleavage in 0X40-triggered pyroptosis of iNKT cells. (A) WT B6 mice were treated with $0 \times 86$ (200 $\mu$ g, i.p.), and 24 hours later iNKT cells in the treated mice were assessed for IL-1 $\beta$ production by intracellular IL-1 $\beta$ staining. Data shown are cells gated on CD1d- $\alpha$ CalCer and CD3 double-positive iNKT cells. The FACS plot shown is representative of one of 6 experiments. (B) ELISA analysis of IL-1 $\beta$ and IL-18 levels in the serum of B6 mice injected with control IgG or OX86 (200 $\mu$ g, i.p.). (C) FACS-sorted iNKT cells from WT B6 and caspase 1-KO mice were stimulated with 0X86 for 24 hours, and cell survival was determined by FACS by staining with the vital dye SYTOX green. Data are from one representative experiment of 6 independent experiments. (D) FACS-sorted iNKT cells from WT B6 mice were stimulated with $0 X 86$ for 24 hours, and LDH in the culture supernatant was assessed using the LDH assay kit. Data represent mean \pm SD of 6 experiments. (E) FACS-sorted iNKT cells from WT B6 mice and caspase 1-KO mice were stimulated with $0 X 86$ for 24 hours, and cleavage of caspase 1 and GSDMD was determined by immunoblotting. $\beta$-Actin was used as a loading control. The blot shown is one of 6 individual experiments. (F) FACS-sorted iNKT cells from WT B6 mice were transduced with a shRNA viral vector targeting GSDMD or a scrambled RNA vector as a control; cells were stimulated with OX86 for 24 hours, and cell survival was determined by FACS following staining with the vital dye SYTOX green. Data shown are from one of 6 independent experiments. (G) The summary graphs represent cell death in relative percentage of gated cells from experiments as described in $\mathbf{F}$. The data shown are mean \pm SD of 6 experiments. The $P$ value was calculated by unpaired 2-tailed Student's $t$ test (B, D, and $\mathbf{G}),{ }^{*} P<0.05$.

OX86 or control IgG, and at various time points we used FACS to examine IL-1 $\beta$ expression by iNKT cells by gating onto the CD1dTet $^{+}$cells. As compared with iNKT cells from control mice, where mature IL-1 $\beta$ was undetectable, those from OX86-treated mice showed robust IL-1 $\beta$ expression, and as many as $42 \%$ iNKT cells stained positive for IL-1 $\beta$ (Figure 3A). As shown by ELISA, OX86 treatment resulted in a marked increase in serum IL-1 and IL-18 levels in the treated mice (Figure 3B). These data are in line with the activation of caspase 1 in iNKT cells by OX40 stimulation. Furthermore, when FACS-sorted iNKT cells from WT B6 mice were stimulated by OX86 in vitro, we observed prominent cell death, as demonstrated by staining with the vital dye SYTOX, whereas iNKT cells from caspase 1-KO mice did not show SYTOX staining after OX86 treatment (Figure 3C), suggesting that caspase 1 also contributes to cell death. In fact, a large increase in the release of lactate dehydrogenase (LDH) into the supernatants by OX40-stimulated iNKT cells suggests a breach of cell membrane integrity and efflux of cellular contents after OX40 stimulation (Figure 3D). To further address the mechanisms of iNKT cell death, we FACS-sorted iNKT cells from WT B6 and caspase 1-KO mice, stimulated them in vitro with OX86, and then examined the cleavage of gasdermin D to its $\mathrm{N}$-domain fragment (32). Immunoblotting showed (Figure 3E) that in iNKT cells from WT B6 mice, stimulation of OX40 with OX86 induced caspase 1 activation, which was associated with cleavage of gasdermin $\mathrm{D}$ to its N-domain fragment, whereas in the absence of OX40 stimulation, neither caspase 1 activation nor gasdermin $\mathrm{D}$ processing occurred in iNKT cells. Interestingly, cleavage of gasdermin D to its active fragment was not observed in iNKT cells from caspase 1KO mice (Figure 3E). To ascertain the role of gasdermin D in iNKT 

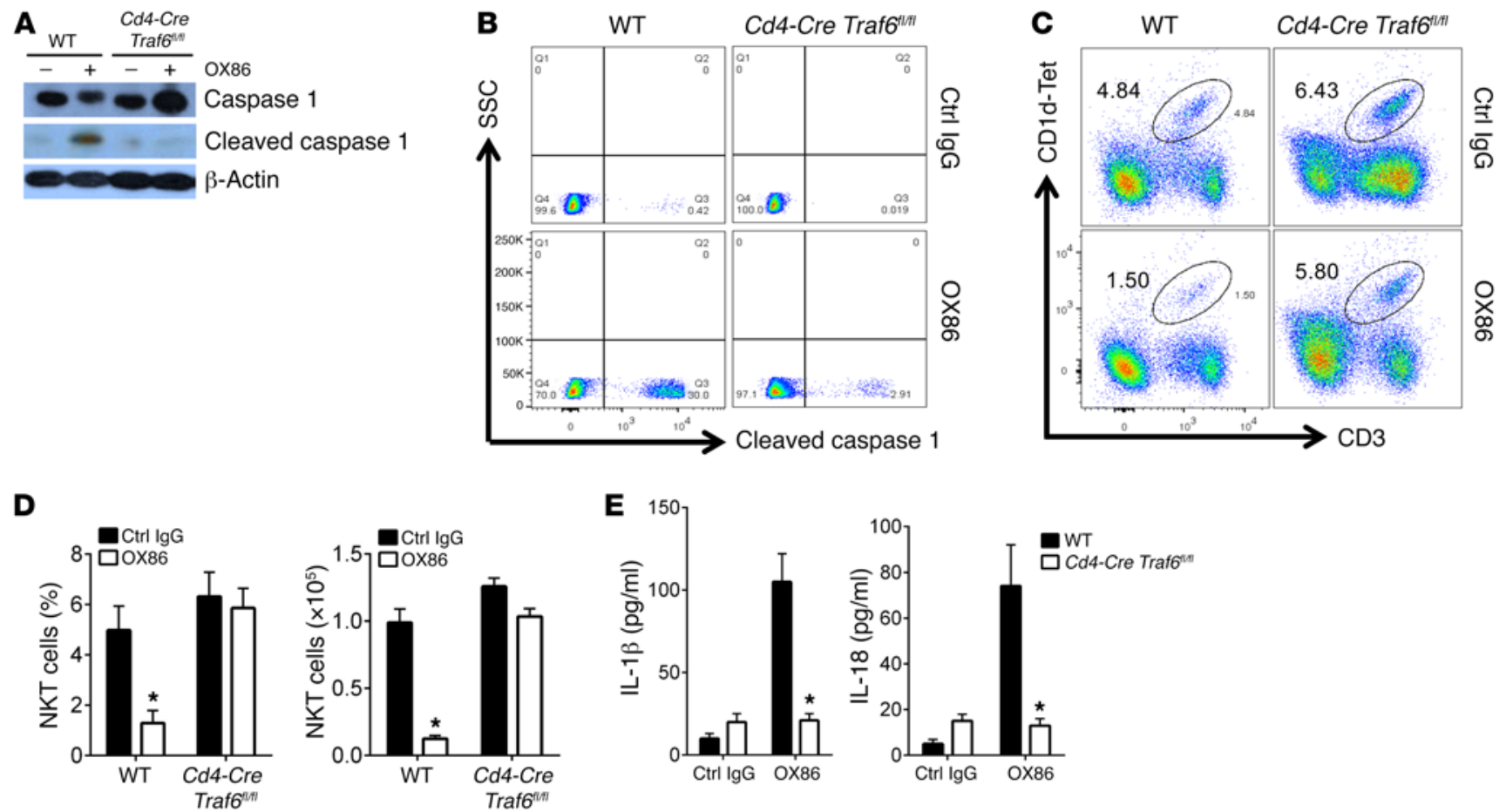

Figure 4. Requirement of TRAF6 in 0X40-mediated activation of caspase 1. (A) FACS-sorted iNKT cells from WT B6 and Cd4-Cre Traff $f^{f / / f l}$ mice were stimulated with 0X86 for 30 minutes, and caspase 1 activation was determined by immunoblotting. $\beta$-Actin was used as a loading control. Data shown represent one of 6 individual experiments. (B) FACS-sorted iNKT cells from WT B6 and Cd4-Cre Traf6 f//fl mice were stimulated with $0 X 86$ for 24 hours, and the induction of active caspase 1 in iNKT cells was determined using a specific staining Ab recognizing cleaved caspase 1 and assessed by FACS. The plot shown represents one of 6 experiments. (C) WT B6 mice and Cd4-Cre Traf6 fl/fl mice were treated with OX86 or a control IgC (200 $\mu$ g, i.p.), and iNKT cells in liver were analyzed by FACS 2 weeks later. The FACS plot shown represents one of 6 independent experiments. (D) The summary graphs represent relative percentage and absolute number of iNKT cells in WT B6 and TRAF6-deleted mice with or without OX86 treatment, as described in C. Data shown are mean \pm SD of 6 mice in each group. (E) ELISA analysis of IL-1 $\beta$ and IL-18 levels in the serum of WT B6 and Cd4-Cre Traf6 f//fl mice treated with control IgG or OX86 (200 $\mu$ g, i.p.). Data represent mean \pm SD of 6 mice in each group. The $P$ value was calculated by unpaired 2-tailed Student's $t$ test between control IgG and $0 \times 86$ treatment $(\mathbf{D}$ and $\mathbf{E}),{ }^{*} P<0.05$.

cell death, we sorted iNKT cells from WT B6 mice and transfected the cells with shRNA to specifically knock down gasdermin D and then examined cell survival following OX40 stimulation. As shown in Figure 3, F and G, knockdown of gasdermin D markedly inhibited iNKT cell death in response to OX40 stimulation. Thus, these data correlate with expanded iNKT cells in caspase 1-KO mice regardless of OX40 stimulation (Figure 3C) and collectively suggest that iNKT cells die of pyroptosis upon OX40 stimulation.

Role of TRAF6-mediated MALT1 recruitment in caspase 1 activation. An outstanding question is how OX40 engagement activates caspase 1 in iNKT cells. The cytoplasmic domain of OX40 does not have any proteolytic activities; it is also incapable of directly interacting with caspase 1 (no consensus binding domain) (34). Thus, the induction of caspase 1 activation must involve other mechanisms. We recently reported that in T cells, OX40 requires TRAF6 to trigger downstream signaling events (18). To examine whether TRAF6 is also involved in iNKT cell depletion, we sorted iNKT cells from WT B6 and Cd4-Cre Traf $6^{\text {t/fl}}$ mice, stimulated them with OX86, and examined caspase 1 activation. As shown in Figure 4A, in TRAF6-deficient iNKT cells OX40 failed to induce caspase 1 activation, as shown by the absence of caspase 1 cleavage by immunoblotting, which is in contrast to the effects of OX40 in WT B6 iNKT cells. The failure of OX40 to activate caspase 1 in
TRAF6-deficient iNKT cells was further confirmed by flow cytometry (Figure 4B). Furthermore, treatment of Cd4-Cre Traf $6^{f / f l}$ mice with OX86 failed to deplete iNKT cells in vivo, whereas the same OX86 protocol resulted in profound iNKT cell depletion in WT B6 mice (Figure 4, C and D). Similarly, as compared with OX86-treated WT B6 mice, treatment of $\mathrm{Cd} 4$-Cre Traf $6^{\sharp / f l}$ mice with OX86 did not result in increased levels of IL-1 $\beta$ and IL-18 in the blood (Figure 4E). These data suggest a critical role for TRAF6 in OX4O-induced activation of caspase 1 and iNKT depletion.

To resolve the molecular mechanisms by which TRAF6 mediates caspase 1 activation, we FACS-sorted WT B6 iNKT cells, stimulated them with OX86 in vitro, and immunoprecipitated them with the anti-TRAF6 mAb. We then examined molecules that potentially interact with TRAF 6 in the immunoprecipitates by immunoblotting assays. As shown in Figure 5A, TRAF6 co-immunoprecipitated with MALT1 in OX40-stimulated iNKT cells, and in this complex BCL10 was also detected. Structurally, MALT1 contains multiple TRAF6-binding sites, and MALT1 additionally contains a death domain, a BLC10-binding site (B1OBS), a caspase-like domain (CaspD), as well as an IKK $\gamma$ binding site (IKBS) (Figure 5B) (35). Functionally, MALT1 can act as a scaffold upon which signaling complexes are assembled or as a paracaspase molecule to mediate proteolytic cleavage of target proteins (35). In order to gain 
A

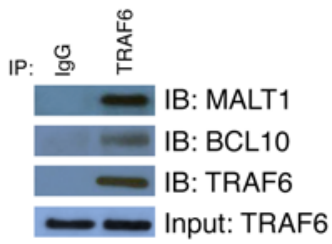

C

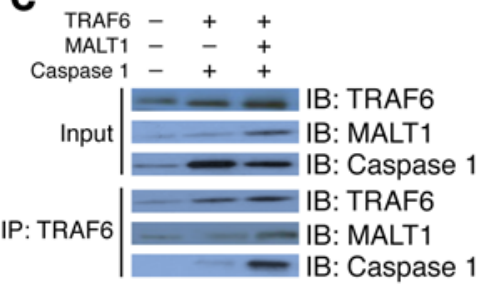

D

TRAF6 binding site
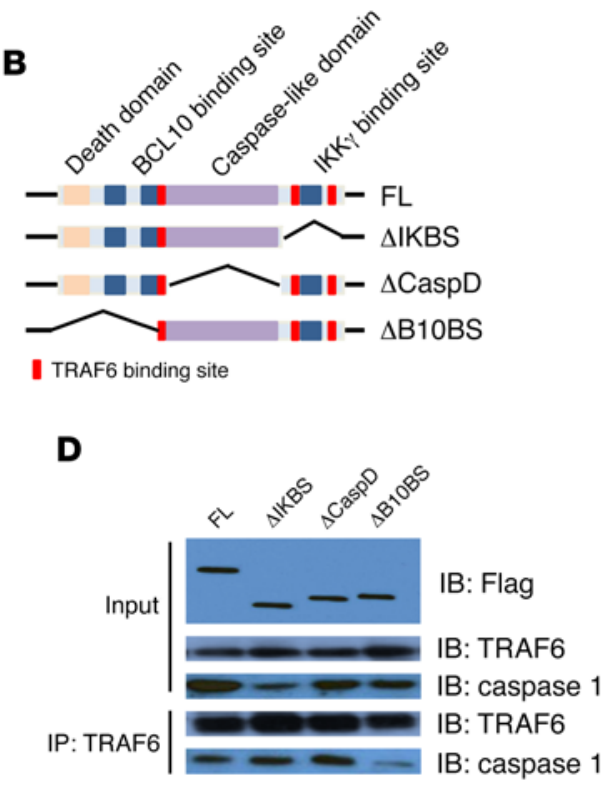

E

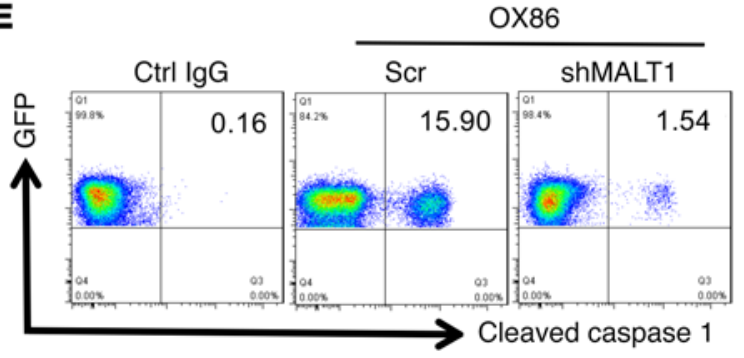

they could be tracked using the anti-FLAG mAb. We transfected the MALT1 mutants into 293T cells and examined caspase 1 recruitment to the MALT1 complex by co-immunoprecipitation assays. As shown in Figure 5D, none of the MALT1 mutations affected the binding of TRAF6 to the MALT1 molecule. However, deletion of B10BS ( $\triangle \mathrm{B} 10 \mathrm{BS})$ abolished the recruitment of caspase 1 into the MALT1TRAF6 complex, whereas deletion of either CaspD ( $\triangle$ CaspD) or IKBS ( $\triangle \mathrm{IKBS})$ did not affect the recruitment of caspase 1 , suggesting that BLC10 is required for recruitment of caspase 1 to the MALT1 complex. We used both RNA knockdown and pharmacological inhibitors to examine the role of the MALT1 paracaspase domain in activation of caspase 1 in iNKT cells. For this purpose, iNKT cells were transfected with retroviral vectors containing either scrambled RNA or MALT1 shRNA. The transduced iNKT cells were marked by GFP expression for analysis. The iNKT cells were then treated with OX86 for 30 minutes, and activation of caspase 1 was determined by flow cytometry. Knockdown of MALT1 markedly inhibited the activation of caspase 1 , as shown by reduced caspase 1 cleavage in flow cytometry (Figure 5E). Furthermore, we stimulated sorted iNKT cells in vitro with OX86, with or without a MALT1 inhibitor (mepazine acetate), and activation of caspase 1 was determined 30

further insights into MALT1 functions, we overexpressed TRAF6, MALT1, and caspase 1 in 293T cells, either individually or in combinations, followed by immunoprecipitation with the anti-TRAF6 mAb. We found that TRAF6 clearly co-immunoprecipitated with MALT1 and caspase 1 in cells transduced with such constructs, but TRAF6 failed to co-immunoprecipitate with caspase 1 in cells without MALT1 (Figure 5C), suggesting that MALT1 is a critical component in TRAF6-mediated activation of caspase 1.

We also made a series of MALT1 mutations, which included deletions of IKBS $(\triangle \mathrm{IKBS})$, CaspD $(\triangle \mathrm{CaspD})$, and B10BS ( $\triangle \mathrm{B} 10 \mathrm{BSS})$. We introduced a FLAG tag to these mutants, so that minutes later. As shown in Figure 5F, the MALT1 inhibitor strongly inhibited the cleavage of caspase 1 in a dose-dependent manner, suggesting the involvement of the MALT1 paracaspase activities in caspase 1 activation. Taken together, these data suggest that in iNKT cells, OX40 ligation recruits MALT1 through TRAF6, and MALT1 then activates caspase 1 to induce pyroptosis of iNKT cells.

Pyroptotic death of $i$ NKT cells in vivo triggers liver injury. We took several approaches to examine the impact of iNKT cell pyroptosis on local inflammation and liver injury. First, we treated $C d 1 d$-KO mice, which are deficient in NKT cells (36), with the anti-OX40 $\mathrm{mAb}$ OX86; 2 weeks later we assessed liver histology, IL-1 $\beta$ and 
A

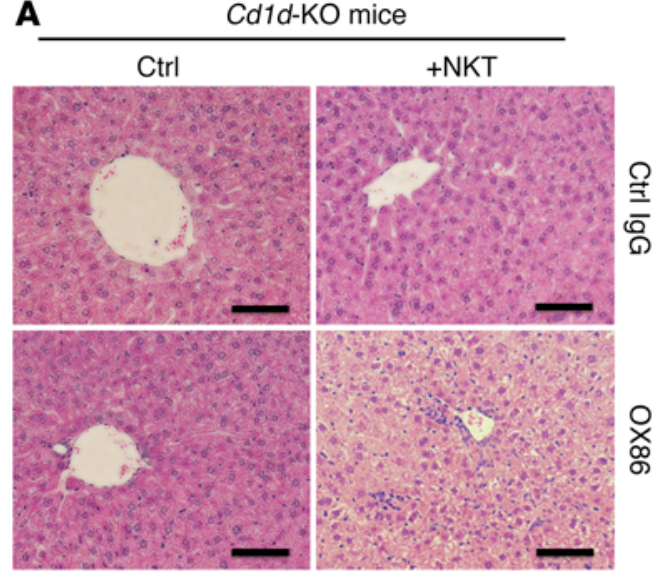

$\mathbf{F}$

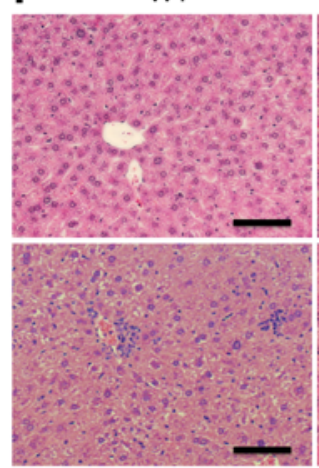

B

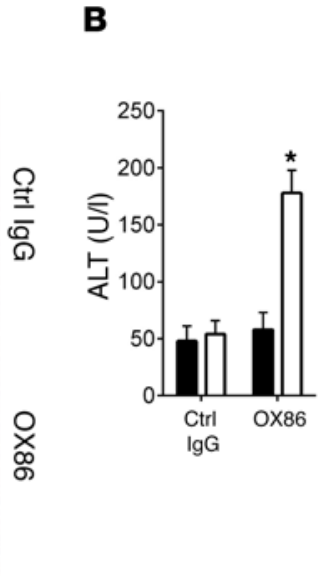

G
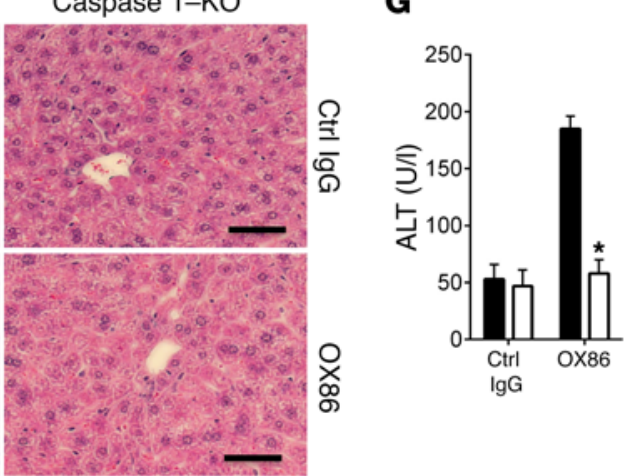

C

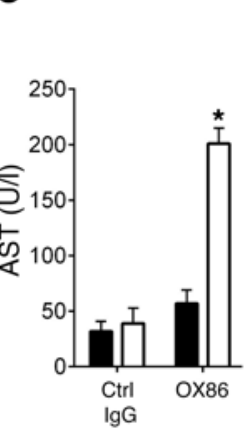

D

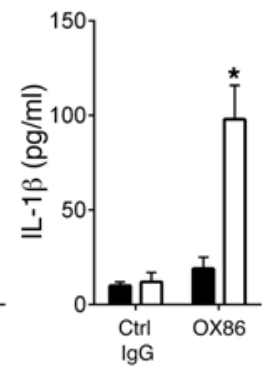

I

H
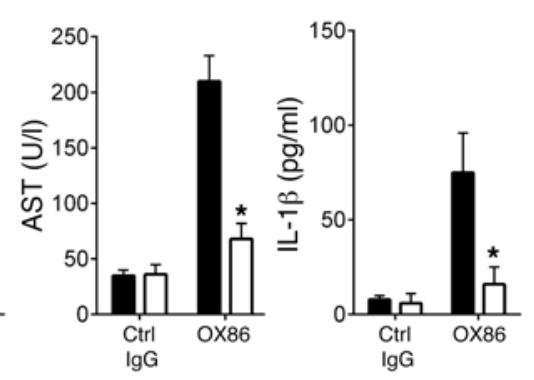

J

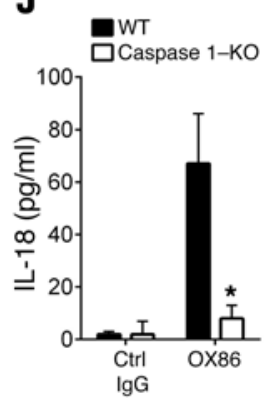

Figure 6. Pyroptotic death of iNKT cells induces liver injury. (A) Liver histology of Cd1d-KO mice and Cd1d-KO mice receiving adoptive transfer of WT B6 iNKT cells and treated with OX86 or a control IgG $(200 \mu \mathrm{g}$, i.p.). The liver pathology of these mice was examined by H\&E staining. The tissue histology is representative of one of 6 experiments. Scale bars: $100 \mu \mathrm{m}$. (B and C) ELISA analysis of serum ALT and AST in Cd1d-KO mice and Cd1d-KO mice receiving transfer of WT iNKT cells and treated with OX86. Data shown are mean \pm SD of 6 mice in each group. (D and E) ELISA analysis of serum IL-1 $\beta$ and IL-18 levels in OX86-treated Cd1d-KO and Cd1d-KO mice receiving transfer of WT B6 iNKT cells. Data shown are mean \pm SD of 6 mice in each group. (F) H\&E sections of liver tissues from WT B6 mice and caspase 1-KO mice treated with control lgG or OX86 mAb (200 $\mu$ g, i.p.), showing liver injury, inflammatory cell infiltrates, and hepatocyte death. The tissue histology shown is representative of one of 6 experiments. Scale bars: $100 \mu \mathrm{m}$. (G and $\mathbf{H})$ ELISA analysis of serum ALT and AST levels in control IgG- and OX86-treated WT B6 and caspase 1-KO mice. Data shown are mean \pm SD of 6 mice in each group. (I and J) ELISA analysis of serum IL-1 $\beta$ and IL-18 levels in control IgG- and OX86-treated WT B6 and caspase 1-KO mice. Bar graphs represent mean \pm SD of 6 mice in each group. $P$ values were calculated by unpaired 2-tailed Student's $t$ test between $C d 1 d-K O$ and $C d 1 d-K O+N K T$ cells (B, C, D, and E) or between WT and caspase 1-KO mice (G, H, I, and $\mathbf{J}),{ }^{*} P<0.05$.

IL-18 production, as well as blood levels of aspartate aminotransferase (AST) and alanine aminotransferase (ALT), enzymes that are associated with hepatocyte injury (37). As shown in Figure $6 \mathrm{~A}$, similar to control IgG-treated mice, treatment of $\mathrm{Cd} 1 \mathrm{~d}$-KO mice with OX86 failed to induce noticeable changes in the liver, as assessed by tissue histology and levels of AST and ALT (Figure 6, B and C). ELISA assay also failed to detect any IL-1 $\beta$ and IL-18 production in the treated $C d 1 d$-knockout mice (Figure 6, D and E). In contrast, in $C d 1 d$-KO mice receiving adoptive transfer of WT B6 iNKT cells, OX86 treatment induced marked liver injury, characterized by inflammatory cell infiltration in the liver, disorganization of hepatic architecture, and stenosis of hepatocytes (Figure 6A). These changes were associated with a rise in liver enzymes (Figure 6, B and C), as well as IL-1 $\beta$ and IL-18 levels in the blood (Figure 6, D and E). These data demonstrate that OX40-induced liver injury clearly requires iNKT cells.

Second, we compared the effects of OX86 treatment on liver injury in WT B6 and caspase 1-KO mice. As shown in Figure 6F, treatment of WT B6 mice with OX86 induced prominent liver injury, which was accompanied by increased AST and ALT levels in the treated mice (Figure 6, G and $\mathrm{H}$ ), as well as increased production of IL-1 $\beta$ and IL-18 (Figure 6, I and J). Interestingly, none of those changes that are associated with liver injury were observed in OX86-treated caspase 1-KO mice (Figure 6, G-J). Thus, OX40 clearly requires caspase 1 to induce liver damage.

Last, we used a concanavalin A-induced (ConA-induced) murine hepatitis model to address the OX40/iNKT cell pathway in liver injury. This model is widely used in the study of liver injury and is known to depend on NKT cells (38). As shown in Figure 7A, injection of a low dose of ConA $(1 \mathrm{mg} / \mathrm{kg})$ into WT B6 mice induced prominent expression of OX40L in the liver, and this was associated with increased serum levels of AST and ALT, as well as increased levels of IL-1 $\beta$ and IL-18 (Figure 7, B-E), confirming the induction of liver injury and expression of OX4OL under inflammatory conditions (13). Interestingly, treatment of the host mice with a blocking anti-OX4OL mAb at the time of ConA injection 

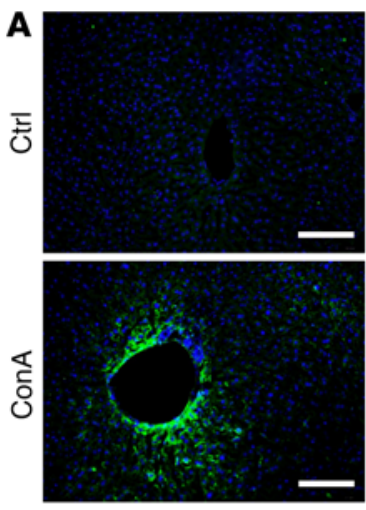

B

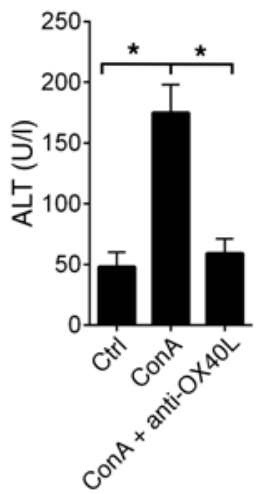

C

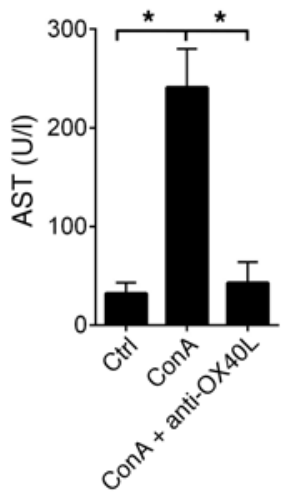

D

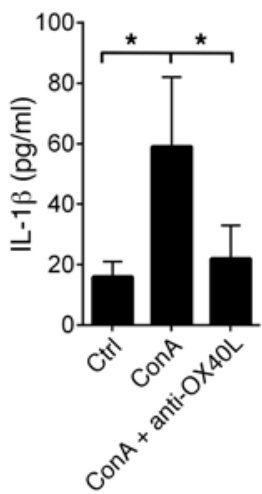

G

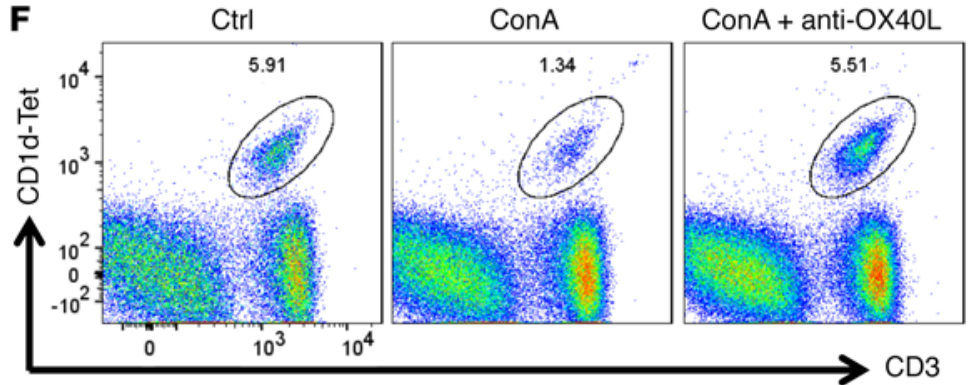

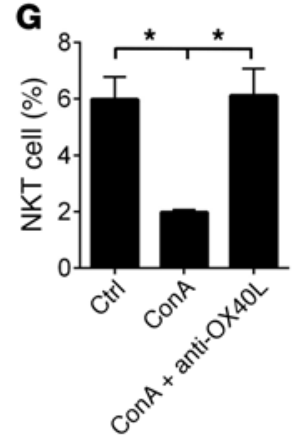

I

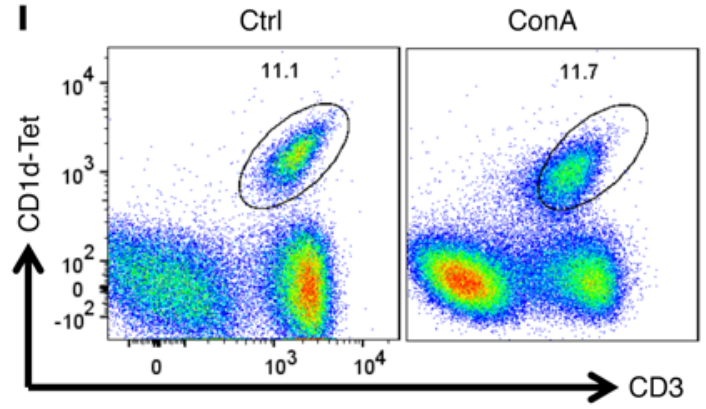

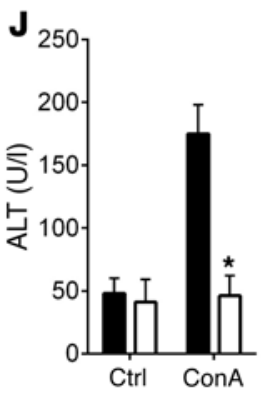

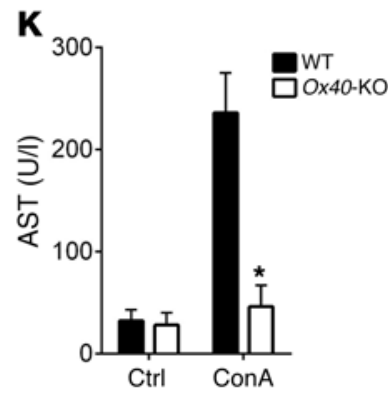

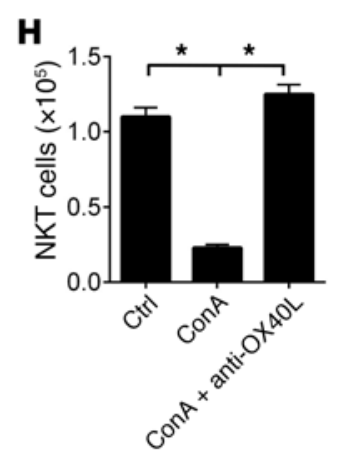

E

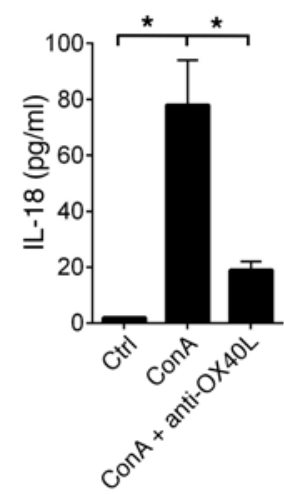

Figure 7. The OX40/NIT pathway is required in ConA-induced hepatitis. (A) The WT B6 mice were injected with ConA at $1 \mathrm{mg} / \mathrm{kg}$, i.p., or PBS as a control, and $\mathrm{OX} 40 \mathrm{~L}$ expression was examined by immunofluorescence staining 1 week later (from 9 fields of view per condition). Cell nuclei were stained with DAPI (blue) for identification and merged with OX4OL (green). Scale bars: $100 \mu \mathrm{m}$. (B and C) ELISA analysis of serum ALT and AST in WT B6 mice injected with ConA, or with ConA and anti-OX4OL antibody. The data shown are mean \pm SD of 6 animals in each group. (D and E) ELISA analysis of serum IL-1 $\beta$ and IL-18 levels in WT B6 mice injected with ConA, or with ConA and anti-OX40L antibody. The data shown are mean \pm SD of 6 animals in each group. (F) WT B6 mice were injected with ConA, and groups of mice were also treated with control IgG or anti-OX40L antibody; iNKT cells in the liver of treated mice were determined by FACS 1 week later. The FACS plot shows representative data of one of 6 animals. ( $\mathbf{G}$ and $\mathbf{H})$ The summary graphs represent relative percentage and absolute number of iNKT cells in WT B6 mice treated with PBS, ConA, or ConA and anti-OX40L mAb. Data shown are mean \pm SD of 6 animals. (I) Ox40-KO mice were injected with ConA or PBS, and iNKT cells in the liver of treated mice were determined by FACS 1 week later. The FACS plot shows representative data of one of 6 animals in each group. (J and $\mathbf{K}$ ) ELISA analysis of serum ALT and AST in Ox4O-KO mice injected with ConA or PBS as a control. The data shown are mean \pm SD of 6 animals in each group. $P$ values were calculated by 1-way ANOVA (B, C, D, E, G, and $\mathbf{H}$ ) and unpaired 2-tailed Student's $t$ test between control and ConA treatment (J and $\mathbf{K}),{ }^{*} P<0.05$.

prevented the induction of AST and ALT, as well as production of the inflammatory cytokine IL-1 $\beta$ and IL-18 (Figure 7, B-E). Flow cytometry showed that ConA substantially reduced iNKT cells in the liver, both in relative percentages and in absolute cell numbers, and this reduction in iNKT cells was completely inhibited by blocking of OX4OL (Figure 7, F-H). Importantly, we found that in Ox4O-KO mice, ConA injection failed to induce liver injury and iNKT depletion (Figure 7, I-K), demonstrating an important role for OX40/NKT cells in liver injury in this model.

\section{Discussion}

In the present study we made several interesting discoveries. First, we demonstrate that, in contrast to its well-publicized role in T cell survival (15), OX40 is a potent death receptor for iNKT cells, and OX40 engagement, either by an agonist mAb or through its ligand (OX4OL), readily depletes iNKT cells in vivo. Second, our data provide the first evidence to our knowledge that besides macrophages, iNKT cells can also commit to pyroptotic cell death, and pyroptosis of iNKT cells exhibits key characteristics similar to 
those of macrophage pyroptosis, including requirement of caspase 1 activation and involvement of gasdermin D cleavage. Moreover, pyroptosis of iNKT cells triggers tissue inflammation and liver injury, thus highlighting that tissue-resident iNKT cells (besides conventional T cells) can contribute to OX40-induced pathology. Third, our study uncovers a molecular mechanism for the activation of caspase 1 , wherein, instead of relying on inflammasome assembly (39), the paracaspase MALT1 provides an alternative mechanism in the activation of caspase 1 (in the absence of danger signals), and in the case of iNKT cells, MALT1 links the costimulatory receptor OX40 to caspase 1-dependent iNKT cell pyroptosis. Finally, the discovery of OX40 in pyroptosis of iNKT cells is significant. Considering the recent report that the OX40/OX4OL pathway is a major driver of tissue fibrosis (23) and the prevalence of liver fibrosis among liver pathologies, our findings may have far-reaching implications in the management of liver diseases.

The finding that iNKT cells can die of pyroptosis is significant, and is especially interesting when considering where they reside and the consequence of the tissue inflammation they produce. We were intrigued that the liver iNKT cells constitutively express high levels of caspase 1 , even under homeostatic conditions; they also express the recently identified pyroptotic protein gasdermin D $(32,33)$, suggesting that the iNKT cells, like macrophages, are likely poised for pyroptotic cell death. Similar to macrophages, pyroptosis of iNKT cells promotes tissue inflammation and tissue damage. But unlike macrophages, pyroptosis of iNKT cells is not induced by activation of the inflammasome, but rather triggered by OX40 stimulation and MALT1 activities. Clearly, the liver is a prime target of iNKT pyroptosis, as tissue injury was not observed in other organs (data not shown). This is consistent with the fact that liver is the primary site for iNKT cells, even though a small proportion of iNKT cells are present outside the liver (4). Our adoptive transfer experiments involving CD1ddeficient hosts demonstrate that iNKT cells alone are sufficient to produce liver damage upon OX40 stimulation. Furthermore, the absence of histological and biochemical evidence of liver damage in OX86-treated CD1d-deficient mice excludes the possibility that other conventional T cells, which are also capable of expressing OX40, are involved in liver injury (14). In OX86-treated mice or ConA-injected mice, we observed extensive infiltration of the liver by inflammatory cells, especially $\mathrm{CD} 11 \mathrm{~b}^{+}$macrophages, but macrophages do not express OX40. Instead, they express OX4OL (13), and their contribution to liver injury may be indirect and secondary to triggering pyroptotic death of iNKT cells. In fact, the finding that in ConA-injected mice blocking the OX40L prevented NKT depletion and liver damage appears to support this notion. Thus, in any clinical situation where expression of OX4OL is potentially upregulated in the liver, pyroptotic death of NKT cells could be triggered, causing liver injury or fibrosis. As liver damage is a significant clinical issue, and frequently observed in different settings, including viral infections and autoimmune diseases (40), the identification of NKT cell pyroptosis in liver damage may have important therapeutic implications.

OX40 is best known for its role in T cell survival and proliferation, as well as in generation of long-lived memory T cells (41-43). Thus, the induction of pyroptotic death of iNKT cells by OX4O signaling is rather unexpected. Mechanistically, we demonstrate that OX40 triggers caspase 1 activation in iNKT cells through recruitment of MALT1, which contains a paracaspase domain traditionally involved in TCR signaling (35). MALT1 is a critical component of the CARMA-BCL10-MALT1 (CBM) complex downstream of the TCR, and in this CBM complex MALT1 can act as a scaffold protein, as well as a proteolytic molecule. In certain models, those two different activities (scaffolding vs. proteolysis) can be segregated in T cells (35). Functionally, the CBM complex plays a key role in activation of the NF- $\kappa \mathrm{B}$ pathway in activated $\mathrm{T}$ cells. In the current study, we found that MALT1 is pivotal in OX40-mediated activation of caspase 1 in iNKT cells, and this process requires TRAF6, which is essential for OX40 signaling (18). In fact, MALT1 has multiple TRAF6 binding sites, and in our models MALT1 clearly co-immunoprecipitates with TRAF6 (Figure 5A), and deletion of Traf6 in iNKT cells prevents caspase 1 activation in response to OX40 stimulation. Furthermore, knockdown of MALT1 in iNKT cells using the RNA interference approach also inhibits OX40-triggered caspase 1 activation (Figure $5 \mathrm{E}$ ). Moreover, the finding that inhibition of the proteolytic activities of MALT1 using chemical inhibitors strongly inhibited the OX40-induced caspase 1 cleavage highlights the importance of its paracaspase activities in OX40-induced NKT pyroptosis (Figure 5F). Collectively, our findings offer strong evidence that besides inflammasome activation, MALT1 provides an alternative means of caspase 1 activation in the induction of pyroptotic cell death. Our data also expand the role of OX40 in the immune system to include not only $\mathrm{T}$ cell survival, but also induction of pyroptotic cell death.

Our study raises several interesting questions that warrant further investigation. Clearly, the underlying mechanisms that induce iNKT cells to constitutively express OX40 and caspase 1 remain unknown. One possibility is that the liver drains the blood from the digestive system, which is rich in food products, metabolites, and constituents of gut microbiota (11), and these elements may contribute to certain features of iNKT cells in the liver. We observed that blocking OX40L or genetic knockout of Ox4O consistently led to an increase in iNKT cells in the liver, and to what extend OX40 regulates iNKT homeostasis under physiological conditions is also unknown. Under inflammatory conditions, however, the intrahepatic myeloid cells do express high levels of OX4OL, which is shown to promote T cell activities (44). Whether this is due to the direct effect of OX40 on T cells or mediated by other extrinsic mechanisms that involve iNKT cells, further studies are warranted. But in the ConA-induced hepatitis model, blocking OX4OL can markedly inhibit liver injury by preventing NKT depletion. Structurally, caspase 1 contains putative sites for MALT1-mediated proteolytic cleavage, but it also contains sites for autocleavage (45), and in the setting of OX40 stimulation, MALT1 is clearly indispensable in caspase 1 activation. Thus, the exact molecular complex assembled downstream of OX40 stimulation in favor of MALT1 activities requires further clarification. Nevertheless, considering the emerging clinical trials of OX40 agonists in the clinic as cancer immunotherapy reagents $(46,47)$, the role of direct OX40 stimulation in pyroptotic death of iNKT cells provides a note of caution on potential liver damage, and further studies in this area will undoubtedly be important in future development of OX40-directed therapies. 


\section{Methods}

Animals. Male WT B6, Cd1d-KO, Cd4-Cre, caspase 1-KO, and DsRed mice were obtained from the Jackson Laboratory. Ox40-KO, OX40L$\mathrm{Tg}$ mice, and Traf6 ${ }^{f / f l}$ mice have been previously described (18). All animals were housed in the specific pathogen-free facility at Houston Methodist Research Institute.

Reagents. Caspase 1 Inhibitor I (sc-358878) and Caspase-3 Inhibitor III (sc-300325) were obtained from Santa Cruz Biotechnology Inc. The MALT1 inhibitor (mepazine acetate), ConA (L7647), and LDH kit were purchased from Sigma-Aldrich.

The anti-mouse OX40 (OX-86) and anti-mouse OX4OL (RM134L) antibodies were obtained from Bio X Cell. The anti-TRAF6 antibody (EP591Y) and anti-caspase 1 (EPR4321) antibodies were from Abcam; and anti- $\beta$-actin (8H10D10), anti-caspase 3 (8G10), antiMALT1, anti-BCL10 (C78F1), anti-rabbit IgG, HRP-linked (catalog 7074) and anti-mouse IgG, HRP-linked antibodies (catalog 7076) were purchased from Cell Signaling Technology. The anti-GSDMD antibody (H-11) was obtained from Santa Cruz Biotechnology Inc. FLAG-TRAF6, p3F-Strep-mMALT1, and pCMV-caspase 1-flag constructs were obtained from Addgene. To construct MALT1 mutants, cDNA from activated T cells was used as a template. The PCR products were digested by restriction enzymes BamHI and EcoRI and then ligated into the pMYs-IRES-GFP vector. All constructs were confirmed by direct sequencing. The primers to generate MALT1 mutants were as follows: for $\triangle \mathrm{B} 10 B \mathrm{BS}$ mutant: forward, 5'-CGGGATCCATGAGGAACCTACTGGTGTCATGT-3', reverse: 5'-GGAATTCCTCAAAACCATGAAGGTCAGTTTTC-3'; for $\triangle \mathrm{IKBS}$ mutant: forward: 5'-CGGGATCCCGCAGTCGAGGCCACCGGCGTA-3', reverse: 5'-GGAATTCCTCTCAGGTGGTTTGTGGACT-3'; for $\triangle$ CaspD mutant: N-terminal fragment, forward: 5'-CGGGATCCCGCAGTCGAGGCCACCGGCGTA-3'，reverse，5'-GGAATTCCACCAGTAGGTTCCTTCATGTTCTA-3'; C-terminal fragment, forward: 5'-CGGGATCCCGAGTCCACAAACCACCTGAGAT-3'; reverse: 5'-GGAATTCCTCAAAACCATGAAGGTCAGTTTTC-3'.

Cell isolation and flow cytometry. The liver from WT B6, Ox40-/, OX4OL-Tg, and Cd4-Cre Traf $6^{f / f l}$ mice were perfused in cold PBS solution in situ and then harvested. Some mice were treated with OX86 or anti-OX40L mAb (200 $\mu$ g, i.p.) before harvesting of liver. The liver was pressed through a $40-\mu \mathrm{m}$ cell strainer (Fisher Scientific) and suspended in RPMI-1640 medium (Gibco BRL) containing 10\% fetal bovine serum. After washing, the lymphocytes were resuspended in $40 \%$ Percoll (GE Healthcare) and centrifuged at $750 \mathrm{~g}$ for 20 minutes at room temperature. The pellet was resuspended in $10 \mathrm{ml} 1 \times \mathrm{RBC}$ Lysis Buffer (eBioscience) and incubated at room temperature for 5 minutes. The cells were then washed and harvested for analysis.

For cell surface staining, cells were stained with APC/Cy7conjugated anti-NK1.1 (PK136; BioLegend), Alexa Fluor 700-conjugated anti-CD3 (17A2; BioLegend), Alexa Fluor 488-conjugated anti-CD19 (6D5; BioLegend), PE-conjugated anti-OX40 (OX86; BioLegend), PE-conjugated anti-CD11b (M1/70; BioLegend), and APC-conjugated CD1d- $\alpha$ GalCer tetramer (NIH Tetramer Core Facility) according to the manufacturers' instructions. For intracellular staining, cells were first fixed and made permeable with Cytofix/Cytoperm solution (BD Pharmingen) and then stained with anti-cleaved caspase 1 antibody (m296; Santa Cruz Biotechnology Inc.) or antiIL-1 $\beta$ antibody (Abcam, catalog ab9722). After 30 minutes, cells were washed twice in washing buffer. The cells were further stained with
PE-conjugated anti-goat secondary IgG (Abcam, catalog ab7004) or PE-conjugated anti-rabbit secondary IgG (poly4064; BioLegend) for 30 minutes and washed 3 times in washing buffer. All samples were acquired with a FACS Fortessa (Beckton Dickinson), and data were analyzed with FlowJo 10 software.

FACS sorting of $i N K T$ cells and in vitro stimulation. Liver was first perfused in situ and pooled, and non-parenchymal cells (mononuclear immune cells) were isolated using Percoll gradients. Cells were stained with CD1d- $\alpha$ GalCer tetramer and anti-CD3 to identify iNKT cells. The iNKT population was then electronically gated, selected, and FACS-sorted with the high-speed cell sorter FACSaria. The sorted iNKT cells were used for immunoblot analysis. In some experiments, the sorted iNKT cells were stimulated with various concentrations of OX86 $(1,2,5,10,20 \mu \mathrm{g} / \mathrm{ml})$, and then harvested and lysed for immunoblot analysis at different time points.

Immunoprecipitation and immunoblotting. iNKT cells from WT B6 and Cd4-Cre Traf6 $6^{f / f l}$ mice, and in some experiments HEK293T cells, were used for transfection experiments to overexpress target proteins. Briefly, cells were transfected with expression plasmids for TRAF6, procaspase 1, MALT1, MALT1 mutants, or a combination of such vectors. Following stimulations, cells were lysed with Pierce IP lysis buffer, and the cell lysates were incubated with Protein A beads for 1 hour (Cell Signaling Technology) to pull down nonspecific binding proteins. After high-speed centrifugation $(13,000 \mathrm{~g})$, the supernatant was incubated with anti-TRAF6 antibody (EP591Y; Abcam) overnight at $4^{\circ} \mathrm{C}$, then Protein A beads were added for another 1 hour of incubation. The protein complexes were pelleted and washed 3 times with IP washing buffer. The immunoprecipitated protein complex was analyzed by immunoblot with anti-TRAF6 and anti-caspase 1 (Abcam). The input cell lysates were also immunoblotted with anti- $\beta$-actin, anti-TRAF6, anti-MALT1, and anti-caspase 1 (Cell Signaling Technology) antibodies. HRP-linked anti-mouse antibodies, or HRP-linked anti-rabbit antibodies were used as secondary antibodies (Cell Signaling Technology). The proteins were detected using the ECL Plus Western blotting detection system.

ELISA, ALT, AST, and LDH assay. The ELISA kits were purchased from eBioscience. IL-1 $\beta$ and IL-18 in serum were measured with the ELISA kits according to the manufacturer's instructions. Serum ALT and AST were measured with an ALT Activity Assay kit and AST Activity Assay kit according to the manufacturer's instructions (SigmaAldrich). LDH released into the culture supernatant was measured by CytoTox 96 Non-Radioactive Cell Viability Assay (Promega).

Retrovirus-mediated gene silencing. The DNA fragments encoding shRNAs targeting mouse MALT1 (GCACTACACGGTTCCATATGT) was synthesized by Integrated DNA Technologies and inserted into pSIREN-RetroQ-ZsGreen1 vector (Promega). The plasmid vector was confirmed by direct sequencing. The plasmids were transfected into packaging Plat-E cells to generate retroviral particles according to the manufacturer's recommendations (Cell Biolabs Inc.). For transduction of iNKT cells with retrovirus, sorted iNKT cells were cultured with CD1d tetramer $(0.2 \mu \mathrm{g} / \mathrm{ml})$ and incubated with freshly prepared retroviral particles in the presence of $10 \mu \mathrm{g} / \mathrm{ml}$ Polybrene (Sigma-Aldrich) by $780 \mathrm{~g}$ centrifugation for 2 hours at $32^{\circ} \mathrm{C}$. Then cells were further cultured for 24 hours at $37^{\circ} \mathrm{C}$ and treated with OX86 for an additional 30 minutes. The cells were harvested and further assessed by FACS.

ConA-induced murine hepatitis model. WT B6 and Ox4O-KO mice were injected i.v. with $1 \mathrm{mg} / \mathrm{kg}$ ConA. Groups of mice were also given 
anti-OX4OL antibody (clone RM134L, $200 \mu \mathrm{g}$ ) administered i.p. after ConA injection, and mice injected with control IgG $(200 \mu \mathrm{g})$ were included as controls. Mice were sacrificed 1 week later, and liver tissue slices were fixed in $10 \%$ formaldehyde solution for histological assessments.

Tissue histopathology. The liver tissues from WT B6, caspase 1$\mathrm{KO}$, and $C d 1 d$-KO mice treated with control IgG or OX86 were prepared and embedded in paraffin. Tissue sections were stained with H\&E. Slides were examined independently, and images were taken with an Olympus microscope.

Statistics. Data are presented as mean \pm SD in each group. Comparison of means in 2 groups was performed using an unpaired 2-tailed Student's $t$ test, and those in 3 groups using 1-way ANOVA test (Prism GraphPad, version 6). A P value less than 0.05 was considered statistically significant.

Study approval. All animal studies were reviewed and approved by the Institutional Committee on Animal Use and Care at Houston Methodist Hospital in Houston Texas in accordance with institutional guidelines.

\section{Author contributions}

PL designed and performed most of the experiments; YZ, YF, XX, $\mathrm{XL}, \mathrm{XZ}$, and YD provided help in the design of some of the key experiments; YC, RMG, NI, and HPM provided key reagents, animal models, and insights. XCL initiated the project, supervised the study, and wrote the manuscript.

\section{Acknowledgments}

We acknowledge the flow cytometry core at Houston Methodist for outstanding services. We thank the NIH Tetramer Core Facility for providing the CD1d tetramer and Laurie Minze for excellent operational support. This work was supported by grants from the NIH (R01AI080779 and R01AI106200).

Address correspondence to: Xian C. Li, Houston Methodist Research Institute, 6670 Bertner Avenue, R7-211, Houston, Texas 77030, USA. Phone: 713.441.2143; E-mail: xcli@houstonmethodist.org.
1. Bendelac A, Savage PB, Teyton L. The biology of NKT cells. Annu Rev Immunol. 2007;25:297-336.

2. Brennan PJ, Brigl M, Brenner MB. Invariant natural killer T cells: an innate activation scheme linked to diverse effector functions. Nat Rev Immunol. 2013;13(2):101-117.

3. Kawano T, et al. CD1d-restricted and TCR-mediated activation of valpha14 NKT cells by glycosylceramides. Science. 1997;278(5343):1626-1629.

4. Godfrey DI, Stankovic S, Baxter AG. Raising the NKT cell family. Nat Immunol. 2010;11(3):197-206.

5. Brennan PJ, et al. Invariant natural killer T cells recognize lipid self antigen induced by microbial danger signals. Nat Immunol. 2011;12(12):1202-1211.

6. Frey AB, Rao TD. NKT cell cytokine imbalance in murine diabetes mellitus. Autoimmunity. 1999;29(3):201-214.

7. Tang ZH, Liang S, Potter J, Jiang X, Mao HQ, Li Z. Tim-3/galectin-9 regulate the homeostasis of hepatic NKT cells in a murine model of nonalcoholic fatty liver disease. J Immunol. 2013;190(4):1788-1796.

8. Cui K, et al. Invariant NKT cells promote alcoholinduced steatohepatitis through interleukin- $1 \beta$ in mice. J Hepatol. 2015;62(6):1311-1318.

9. Wang H, Feng D, Park O, Yin S, Gao B. Invariant NKT cell activation induces neutrophil accumulation and hepatitis: opposite regulation by IL-4 and IFN- $\gamma$. Hepatology. 2013;58(4):1474-1485.

10. Santodomingo-Garzon T, Swain MG. Role of NKT cells in autoimmune liver disease. Autoimmun Rev. 2011;10(12):793-800.

11. Robinson MW, Harmon C, O'Farrelly C. Liver immunology and its role in inflammation and homeostasis. Cell Mol Immunol. 2016;13(3):267-276

12. Kronenberg M. Toward an understanding of NKT cell biology: progress and paradoxes. Annu Rev Immunol. 2005;23:877-900.

13. Croft M. Control of immunity by the TNFRrelated molecule OX40 (CD134). Аnпи Rev Immunol. 2010;28:57-78.

14. Li XC, Rothstein DM, Sayegh MH. Costimulatory pathways in transplantation: challenges and new developments. Immunol Rev. 2009;229(1):271-293.

15. Rogers PR, Song J, Gramaglia I, Killeen N, Croft
M. OX40 promotes Bcl-xL and Bcl-2 expression and is essential for long-term survival of CD4 T cells. Immunity. 2001;15(3):445-455.

16. Kopf M, et al. OX40-deficient mice are defective in Th cell proliferation but are competent in generating $B$ cell and CTL Responses after virus infection. Immunity. 1999;11(6):699-708.

17. Ito $\mathrm{T}$, et al. TSLP-activated dendritic cells induce an inflammatory $\mathrm{T}$ helper type 2 cell response through OX40 ligand. JExp Med. 2005;202(9):1213-1223.

18. Xiao $\mathrm{X}$, et al. OX40 signaling favors the induction of $\mathrm{T}(\mathrm{H}) 9$ cells and airway inflammation. $\mathrm{Nat}$ Immunol. 2012;13(10):981-990.

19. Vu MD, et al. OX40 costimulation turns off Foxp3+ Tregs. Blood. 2007;110(7):2501-2510.

20. Piconese S, Valzasina B, Colombo MP. OX40 triggering blocks suppression by regulatory $\mathrm{T}$ cells and facilitates tumor rejection. J Exp Med. 2008;205(4):825-839.

21. Murata K, Nose M, Ndhlovu LC, Sato T, Sugamura K, Ishii N. Constitutive OX40/OX40 ligand interaction induces autoimmune-like diseases. JImmunol. 2002;169(8):4628-4636.

22. Xiao X, et al. New insights on OX40 in the control of $\mathrm{T}$ cell immunity and immune tolerance in vivo. J Immunol. 2012;188(2):892-901.

23. Elhai M, et al. OX4OL blockade protects against inflammation-driven fibrosis. Proc Natl Acad Sci U S A. 2016;113(27):E3901-E3910.

24. Zaini J, et al. OX40 ligand expressed by DCs costimulates NKT and CD4+ Th cell antitumor immunity in mice. J Clin Invest. 2007;117(11):3330-3338.

25. Diana J, et al. NKT cell-plasmacytoid dendritic cell cooperation via OX40 controls viral infection in a tissue-specific manner. Immunity. 2009;30(2):289-299.

26. Damayanti $T$, et al. Serial OX40 engagement on $\mathrm{CD} 4+\mathrm{T}$ cells and natural killer $\mathrm{T}$ cells causes allergic airway inflammation. Am J Respir Crit Care Med. 2010;181(7):688-698.

27. Fan Z, et al. In vivo tracking of 'color-coded' effector, natural and induced regulatory $\mathrm{T}$ cells in the allograft response. Nat Med.2010;16(6):718-722.

28. Yuan J, Najafov A, Py BF. Roles of caspases in necrotic cell death. Cell. 2016;167(7):1693-1704.

29. Ashkenazi A, Salvesen G. Regulated cell death: signaling and mechanisms. Annu Rev Cell Dev Biol. 2014;30:337-356.

30. Doitsh G, et al. Cell death by pyroptosis drives CD4 T-cell depletion in HIV-1 infection. Nature. 2014;505(7484):509-514.

31. Shalini S, Dorstyn L, Dawar S, Kumar S. Old, new and emerging functions of caspases. Cell Death Differ. 2015;22(4):526-539.

32. Shi J, et al. Cleavage of GSDMD by inflammatory caspases determines pyroptotic cell death. Nature. 2015;526(7575):660-665.

33. Kayagaki N, et al. Caspase-11 cleaves gasdermin D for non-canonical inflammasome signalling. Nature. 2015;526(7575):666-671.

34. Croft M. Co-stimulatory members of the TNFR family: keys to effective T-cell immunity? Nat Rev Immunol. 2003;3(8):609-620.

35. Thome M. Multifunctional roles for MALT1 in T-cell activation. Nat Rev Immunol. 2008;8(7):495-500.

36. Teige A, Bockermann R, Hasan M, Olofsson KE, Liu Y, Issazadeh-Navikas S. CD1d-dependent NKT cells play a protective role in acute and chronic arthritis models by ameliorating antigen-specific Th1 responses. JImmunol. 2010;185(1):345-356.

37. Kim WR, Flamm SL, Di Bisceglie AM, Bodenheimer HC, Public Policy Committee of the American Association for the Study of Liver Disease. Serum activity of alanine aminotransferase (ALT) as an indicator of health and disease. Hepatology. 2008;47(4):1363-1370.

38. Takeda K, Hayakawa Y, Van Kaer L, Matsuda H, Yagita H, Okumura K. Critical contribution of liver natural killer $\mathrm{T}$ cells to a murine model of hepatitis. Proc Natl Acad Sci U S A. 2000;97(10):5498-5503.

39. Miao EA, et al. Caspase-1-induced pyroptosis is an innate immune effector mechanism against intracellular bacteria. Nat Immunol. 2010;11(12):1136-1142.

40. Swain MG. Fatigue in liver disease: pathophysiology and clinical management. Can J Gastroenterol. 2006;20(3):181-188. 
41. Weinberg AD. The role of OX40 (CD134) in T-cell memory generation. Adv Exp Med Biol. 2010;684:57-68.

42. Ruby CE, Redmond WL, Haley D, Weinberg AD. Anti-OX40 stimulation in vivo enhances CD8+ memory $\mathrm{T}$ cell survival and significantly increases recall responses. Eur JImmunol. 2007;37(1):157-166.

43. Gaspal FM, Kim MY, McConnell FM, Raykundalia C, Bekiaris V, Lane PJ. Mice deficient in
OX40 and CD30 signals lack memory antibody responses because of deficient $\mathrm{CD} 4 \mathrm{~T}$ cell memory. JImmunol. 2005;174(7):3891-3896.

44. Huang LR, et al. Intrahepatic myeloid-cell aggregates enable local proliferation of $\mathrm{CD} 8(+)$ $\mathrm{T}$ cells and successful immunotherapy against chronic viral liver infection. Nat Immunol. 2013;14(6):574-583.

45. Broz P, von Moltke J, Jones JW, Vance RE, Monack DM. Differential requirement for caspase-1 autoproteolysis in pathogen-induced cell death and cytokine processing. Cell Host Microbe. 2010;8(6):471-483.

46. Sugamura K, Ishii N, Weinberg AD. Therapeutic targeting of the effector T-cell co-stimulatory molecule OX40. Nat Rev Immunol. 2004;4(6):420-431.

47. Jensen SM, et al. Signaling through OX40 enhances antitumor immunity. Semin Oncol. 2010;37(5):524-532. 